\title{
Non-Hermitian quantum theory with maximal mass
}

\author{
Rodionov Vasilij N. ${ }^{1, \star}$ and Kravtsova Galina A. ${ }^{2, \star \star}$ \\ ${ }^{1}$ Plekhanov Russian University of Economics \\ ${ }^{2}$ Moscow State University
}

\begin{abstract}
Non-Hermitian fermion Hamiltonian with $\gamma_{5}$ mass extension is studied. The connection of this $\mathcal{P} \mathcal{T}$-symmetrical pseudo-Hermitian model with the geometrical theory with maximal mass is established. The interaction of modified Dirac particles with the external magnetic field is considered. In particular, modified neutrinos is under studying. The results of neutrinos mass values measurements are explained.
\end{abstract}

\section{Introduction}

It is well known fact that the most important recent experimental data are the following:

1). approximately only $5 \%$ of the mass density of the Universe is consistent with the mass of known particles;

2). neutrinos have a nonzero mass.

This new knowledge put serious questions for investigators. The Standard Model can not present the cardinal decision of these problems. Here we suggest to find this decision with the help of socalled theory with the maximal mass. We suppose that this theory will can become something like "Standard Model in the curved p-space". But this is a far future, now we study the simple variant of this model.

It is possible to say that the theory with the maximal mass consists of two parts: Geometrical Theory and Algebraic one. We should consider shortly both of them here.

\section{Geometrical Theory with Maximal Mass}

The history of theory with maximal mass began in 1965, when Markov suggested a hypothesis that mass spectrum of elementary particles should be cut off at the Planck mass $M_{\text {Planck }} \approx 10^{19} \mathrm{GeV}$ [1]. He named the particle with the maximal mass maximon.

The next step was made by Kadyshevsky in 1978. He proposed "geometrical approach" for building of the theory [2]. It means that Minkowski planar 4-dimensional $p$-space is replaced by the curved momentum 4-space which is the hyperboloid surface in a 5-dimensional $p$-space,

$$
p_{0}^{2}-p_{1}^{2}-p_{2}^{2}-p_{3}^{2}+p_{5}^{2}=M^{2},
$$

${ }^{\star}$ e-mail: rodyvn@mail.ru

$\star \star$ e-mail: gakr@chtc.ru 
where $M$ is the curvature radius of the 5-dimensional hyperboloid. It is called the space of anti-de Sitter. Note, that in the approximation

$$
\left|p_{0}\right|,|\vec{p}| \ll M, p_{5} \cong M
$$

anti-de Sitter geometry is transformed into Minkowski geometry in the 4-dimensional pseudoEuclidean $p$-space (the so called "planar limit").

The postulate that the objects with masses $m$ larger than $M$ cannot be considered as elementary particles, since no local fields correspond to them, is accepted:

$$
m \leq M
$$

In this theory it becomes the fundamental principle together with the ordinary postulates of quantum theory. The parameter $M$ is named maximal mass here. It corresponds to the mass of maximon.

Note, that for a free particle, $p_{0}^{2}-\vec{p}^{2}=m^{2}$, condition (3) is automatically satisfied on surface (1).

The development of this theory ([3]-[14]) leads to the following facts. One of the main features of the Geometrical Theory fermion sector is that the Dirac operator becomes modified [3],

$$
\mathcal{D}\left(p, p_{5}\right) \equiv p_{n} \gamma^{n}-\left(p_{5}-M\right) \gamma^{5}+2 M \sin \frac{\mu}{2},
$$

where $\cos \mu=\sqrt{1-\frac{m^{2}}{M^{2}}}$. Easy to see, that in "planar limit" $M \rightarrow \infty$ (see also (2)) modified $D\left(p, p_{5}\right)$ becomes ordinary Dirac operator $D$. Modified equations of motion corresponding to operator (4) in the Hamiltonian form are the following,

$$
\left(p_{0}-\hat{\alpha} \mathbf{p}-\hat{\beta} m_{1}-\hat{\beta} \gamma^{5} m_{2}\right) \Psi\left(x, t, x_{5}\right)=0 .
$$

Here the matrices $\hat{\beta}=\gamma_{0}, \gamma^{i}=\hat{\beta} \hat{\alpha}^{i}$, and we have notation

$$
m_{1}=2 M \sin \mu / 2, \quad m_{2}=2 M \sin ^{2} \mu / 2,
$$

and $p_{\mu}=i \partial_{\mu}$.

The another specific feature of Geometrical Theory is the existence of so-called the exotic Dirac operator in it [3]. It has the following form,

$$
D_{\text {exotic }}(p, M)=p_{\nu} \gamma^{v}+\left(p_{5}+M\right) \gamma^{5}-2 M \cos (\mu / 2)
$$

and the corresponding equation of motion

$$
\left(p_{0}-\hat{\alpha} \mathbf{p}-\hat{\beta} m_{3}-\hat{\beta} \gamma^{5} m_{4}\right) \Psi^{e x}\left(x, t, x_{5}\right)=0
$$

where $m_{3}=2 M \cos \mu / 2, m_{4}=2 M \cos ^{2} \mu / 2$. In planar limit exotic operator and exotic equation don't come to ordinary Dirac operator and Dirac equation of motion. So one can propose that they associated with some fermion fields that has no analogues in Standard Model. They were named as exotic fermions. It is proposed that they put a contribution to the Dark Matter in the Universe.

In the quantum mechanical approximation, the Hamiltonians corresponding to modified equations of motion for ordinary and exotic fermion fields can be represented in the following form:

$$
\hat{H}=\overrightarrow{\hat{\alpha}} \vec{p}+\hat{\beta}\left(m_{1}+m_{2} \gamma_{5}\right),
$$




$$
\hat{H}_{\text {exotic }}=\overrightarrow{\hat{\alpha}} \vec{p}+\hat{\beta}\left(m_{3}+m_{4} \gamma_{5}\right) \text {. }
$$

Easy to see that these expressions turn out to be non-Hermitian due to the $\gamma_{5}$-mass terms. In summary, we can conclude that the Geometric Theory leads to the emergence of non-Hermitian Lagrangian in the fermion sector. It seems to be crucial point of the theory, but the solution of the problem was found.

\section{Algebraic Theory with Maximal Mass}

At the end of 90th and in the early 2000-ies a group of mathematicians found a solution to this problem. They proved that if the model isn't Hermitian but is PT-symmetrical [15], [16] or pseudoHermitian $\left(\eta_{0} \mathrm{H}_{0}^{-1}=\mathrm{H}^{\dagger}, \quad \eta_{0}\right.$ is linear Hermitian operator $)$ [17], so the average of Hamiltonian can become real in new scalar product, which is modified due to special operator $\mathrm{C}$. The formula for this operator was found. This method appears to be very powerful, so such models become very popular (see, for example, [18]-[37]).

So it is possible to apply this technique for investigation of non-Hermitian fermionic model which arises in Geometrical Theory with Maximal Mass.

Let's try to study our Hamiltonian (9) at the algebraic theory point of view. ${ }^{1}$ We'll call it the Algebraic Theory with the physical mass (mass of the particle) $m^{2}=m_{1}^{2}-m_{2}^{2}$. One can easily see that these Hamiltonians are not Hermitian, but PT -symmetrical and pseudo-Hermitian with

$$
\eta_{0}=e^{\alpha \gamma_{5}}, \quad \text { where } \alpha \text { is defined as } \quad m_{1}=m \operatorname{ch} \alpha ; \quad m_{2}=m \operatorname{sh} \alpha
$$

(see [38], [39], [40]). It is so-called non-disturbed PT-symmetry in the theory when $m$ is real, t.e. $m_{1}^{2} \geq m_{2}^{2}$.

Very important feature of Algebraic Theory is that there exists a parameter of maximal mass in it. Indeed, due to simple mathematical manipulations one can find ([39], [40]), that

$$
m \leq \frac{m_{1}^{2}}{2 m_{2}} \equiv m_{\max }
$$

This formal estimation becomes the physical one due to the postulate which we propose: $m_{\max }$ must be equal to $M$ in Geometrical Theory. So these two theories become equivalent (at least, on this simple level). This fact allows to Algebraic Theory to find a physical sense, and to Geometrical Theory to solve the problem of non-Hermitian Hamiltonian in fermion sector. But for understanding this equality it is necessary to put the postulate $m_{\max }=M$ in the theory for all particles [39], [40]. In this case $M$ have to be maximal mass for all the elementary particles mass spectrum.

Due to parametrization (11) the parameters $m_{1}, m_{2}$ can be re-expressed through another two ones: $m$ and $m_{\max }$,

$$
\begin{gathered}
m_{1}^{\mp}=\sqrt{2} m_{\max } \sqrt{1 \mp \sqrt{1-m^{2} / m_{\text {max }}^{2}}} ; \\
m_{2}^{\mp}=m_{\max }\left(1 \mp \sqrt{1-m^{2} / m_{\text {max }}^{2}}\right) .
\end{gathered}
$$

So if we know the physical mass of a particle and the mass of maximon, we can calculate the parameters $m_{1}, m_{2}$ for Hamiltonian (9). But obviously we have two values of the parameters $m_{1}, m_{2}$ for

\footnotetext{
${ }^{1}$ See also [27]
} 
each value of $m$. It means that in Algebraic Theory exotic particles also arise, such as in Geometrical one [40]. The high sign in formulas (13), (14) corresponds to ordinary particles, and the down sign to exotic fermions. This fact is the indirect confirmation of the equality Geometrical (in fermion sector) and Algebraic Theories. Even more, if in the formulas (13), (14) we use $M$ instead of $m_{\max }$, we get the correspondence (equality) of the parameters in Algebraic and Geometric Theories,

$$
m_{1}^{-} \leftrightarrow m_{1}, \quad m_{1}^{+} \leftrightarrow m_{3}, \quad m_{2}^{-} \leftrightarrow m_{2}, \quad m_{2}^{+} \leftrightarrow m_{4} .
$$

As we note above, the exotic particles are the candidates for Dark Matter in the Universe. Of course, maximon have to put contribution to Dark Matter too.

Easy to see also that modified Dirac Hamiltonian $\hat{H}$ in algebraic theory with maximal mass and ordinary Dirac Hamiltonian $\hat{H}_{0}$ are connected by the following transformations,

$$
\hat{H}_{0}=\eta \hat{H} \eta^{-1}, \quad \hat{H}_{0}=\eta^{-1} \hat{H}^{\dagger} \eta, \quad \eta=e^{\alpha \gamma_{5} / 2}
$$

(see [38], [39], [41]). Note that $\eta$ isn't unitary, so this modified Dirac model and ordinary Dirac model are not equivalent. ${ }^{2}$

Using a common algebraic theory [17], one can find C-operator for the definition of modified scalar product,

$$
C=\eta_{0}^{-1} \mathcal{P}=e^{-\alpha \gamma_{5}} \gamma_{0} .
$$

It is possible to demonstrate that the new scalar product is positive defined and the average values of the Hamiltonian (9), (10) are real [40].

Also the eigenvalues and eigenvectors for free modified Dirac Hamiltonian were found [40], [43], [44]. The law of the current density continuity was obtained [45].

All these problems were solved for the algebraic model with maximal mass, where fermionic fields interact with the external magnetic field. Namely, let's study modified fermion without spin in the homogeneous magnetic field $\overrightarrow{\mathcal{H}}=(0,0, \mathcal{H})$. The electromagnetic field potentials in gauge [46] can be chosen in the following form: $A_{0}=0, A_{1}=0, A_{2}=\mathcal{H} x_{1}, A_{3}=0$. Considering the equation

$$
H \widetilde{\psi}=E \widetilde{\psi},
$$

where

$$
H=(\vec{\alpha} \overrightarrow{\mathcal{P}})+\beta m_{1}+\beta \gamma_{5} m_{2},
$$

and $\mathcal{P}_{\mu}=i \partial_{\mu}-e A_{\mu} ; e=-|e|$, one can find ([45], [47], [48], [49], see also review [50])

$$
E= \pm \sqrt{m_{1}^{2}-m_{2}^{2}+2 \gamma n+p_{3}^{2}}
$$

where $n=0,1,2 \ldots$ and $\gamma=|e| \mathcal{H}$. Taking into account that $m^{2}=m_{1}{ }^{2}-m_{2}{ }^{2}$, we can see that this result coincides with the eigenvalues of the Hermitian Hamiltonian describing Landau relativistic levels (see, e.g., [46]).

The situation is cardinally changed when the spin of the fermion is taken into account. We consider the Hamiltonian [47], [48], [51]

$$
H_{\Delta \mu}=\vec{\alpha} \overrightarrow{\mathcal{P}}+\beta\left(m_{1}+\gamma_{5} m_{2}\right)+\Delta \mu \beta(\vec{\sigma} \overrightarrow{\mathcal{H}}),
$$

where $\Delta \mu=\left(\mu-\mu_{0}\right)=\mu_{0}(g-2) / 2$. Here, $\mu$ is the magnetic moment of the fermion, $g$ is the gyromagnetic factor of the fermion, $\mu_{0}=|e| / 2 m$ is the Bohr magneton, and and $\sigma^{\mu \nu}=i / 2\left(\gamma^{\mu} \gamma^{\nu}-\gamma^{v} \gamma^{\mu}\right)$.

\footnotetext{
${ }^{2}$ See, for comparison, [42].
} 
Thus, the phenomenological constant $\Delta \mu$ introduced by Pauli is a part of the equation and can be interpreted from the point of view of the quantum field theory. Taking into account the quantum electrodynamic contribution into the anomalous magnetic moment of the electron to $e^{2}$, we have $\Delta \mu=\frac{\alpha}{2 \pi} \mu_{0}$, where $\alpha=e^{2}=1 / 137$ is the fine structure constant, and it is still assumed that the external field potential satisfies the free Maxwell equation. As it was shown in paper [45], [48], with the help of the modified Dirac-Pauli equation we can find the exact values of the energy spectrum for the Hamiltonian (21),

$$
E\left(\zeta, p_{3}, 2 \gamma n, \mathcal{H}\right)= \pm \sqrt{p_{3}^{2}-m_{2}^{2}+\left[\sqrt{m_{1}^{2}+2 \gamma n}+\zeta \Delta \mu \mathcal{H}\right]^{2}}
$$

where $\zeta= \pm 1$ characterizes the fermion spin projection onto the magnetic field direction.

For the case of neutral particles with anomalous magnetic moment it is necessary to replace the value of the quantized transverse momentum of a charged particle in a magnetic field by the regular value, $2 \gamma n \rightarrow p_{1}^{2}+p_{2}{ }^{2}=p_{\perp}{ }^{2}$. As a result, we obtain [47], [51]

$$
E\left(\zeta, p_{3}, p_{\perp}, \mathcal{H}\right)= \pm \sqrt{p_{3}^{2}-m_{2}^{2}+\left[\sqrt{m_{1}^{2}+p_{\perp}^{2}}+\zeta \Delta \mu \mathcal{H}\right]^{2}}
$$

Namely, the estimations were found for the neutrinos ([40], [50]), [52].

Formulas (22), (23) show that taking into account of the spin with the magnetic field interaction allows us to separate the variables $m_{1}$ and $m_{2}\left(m_{1}^{\mp}\right.$ and $\left.m_{2}^{\mp}\right)$, and therefore, separate ordinary and exotic particles. This means that for the observation of exotic particles it is not necessary ultra-high energy. It is necessary the presence of the anomalous magnetic moment of the particles and it's interaction with the magnetic field. It's very important to emphasize that the experiment, in which the exotic fermions may be observable, proves the existence of Maximal mass.

\section{Algebraic Theory and Experiment}

The neutrinos mass observation may become experiment mentioned above [53]. Just the existence of the exotic particles in the theory allows to explain the experiments for the neutrino mass observations. Really, using (23) one could write for neutrino mass square in the magnetic field:

$$
\tilde{m}_{v}^{2}(\mathcal{H})=\tilde{m}_{v}^{2} \pm 4 \Delta \mu \mathcal{H} M
$$

We want draw attention to the fact that we deal with experiment measurements of neutrinos mass with taking into account influence of the magnetic fields. The result of experiment depends on the spin direction relatively magnetic field direction, and the exotic neutrinos make the principal contribution.

According to scheme of the the Troitsk experimental installation for measurement of mass the electron antineutrino gas-tritium is injected into a long tube, where a strong magnetic field (up to $\mathrm{H}=$ $0.8 \mathrm{~T}=8000 \mathrm{Gauss}$ ) is existed [54], [55]. The results of Troitsky experiment at 95\% confidence (C.L.) are the following [56]

$$
m_{v}^{2}=-0.67 \pm 1.89_{\text {stat }} \pm 1.68_{\text {syst }} e V^{2} .
$$

If we suggest that the beam of neutrinos partially consist of pseudo-Hermitian components exotic neutrinos $\left(\tilde{m}_{v}^{2}\right)$ that we can obtain the following evaluation $\tilde{m}_{v}^{2}(\mathcal{H})=\tilde{m}_{v}^{2}-4 \Delta \mu \mathcal{H} M$. If we consider estimation, which follows from the ordinary experimental dates $\tilde{m}_{v}=m_{v}=1 \mathrm{eV}$ and suppose $\tilde{m}_{v}^{2}(\mathcal{H})=$ $-1 e V^{2}$ so we can evaluate that $4 \Delta \mu \mathcal{H} M \cong 2 e V^{2}$ and moreover, estimate $M \cong 2 \cdot 10^{14}-10^{15} \mathrm{GeV}$. 


\section{Conclusions}

In summary, we can conclude that the Algebraic Theory makes possible to describe the experimental data of the mass squared neutrinos observations and to estimate the value of maximal mass $M$ in the low energies region.

So the experiments for observations of mass squared neutrinos may prove the existence of exotic particles and confirm the restriction of elementary particles mass spectrum by the maximal mass $M$.

\section{References}

[1] M.A.Markov, Prog. Theor Phys. Suppl., Commemoration Issue for the Thirtieth Anniversary of Meson Theory and Dr. H. Yukawa, p. 85 (1965); Sov. Phys. JETP, 24, p. 584 (1967).

[2] V.G.Kadyshevsky, Nucl. Phys., B141, p 477 (1978); in Proceedings of International Integrative Conference on Group theory and Mathematical Physics, Austin, Texas, 1978; Fermilab-Pub. 78/70THY, Sept. 1978; Phys. Elem. Chast. Atom. Yadra, 11, p.5 (1980).

[3] V.G.Kadyshevsky, M.D.Mateev, V.N.Rodionov, A.S.Sorin, Towards a maximal mass model. CERN TH/2007-150; hep-ph/0708.4205.

[4] V.G.Kadyshevsky, M.D.Mateev, V.N.Rodionov, A.S.Sorin, Doklady Physics 51, p.287 (2006); hep-ph/0512332.

[5] V.G.Kadyshevsky, Phys. Part. Nucl., 29, p.227 (1998).

[6] V.G.Kadyshevsky, M.D.Mateev, Phys. Lett., B106, p.139 (1981).

[7] V.G.Kadyshevsky, M.D.Mateev, Nuovo Cimento, A87, p.324 (1985).

[8] M.V.Chizhov, A.D.Donkov, V.G.Kadyshevsky, M.D.Mateev, Nuovo Cimento, A87, p.350 (1985).

[9] M.V.Chizhov, A.D.Donkov, V.G.Kadyshevsky, M.D.Mateev, Nuovo Cimento, A87, p.373, (1985).

[10] V.G.Kadyshevsky, Phys. Part. Nucl., 29, p.227 (1998).

[11] V.G.Kadyshevsky, D.V.Fursaev, JINR Rapid Commun., N 6, p.5 (1992).

[12] R.M.Ibadov, V.G.Kadyshevsky, Preprint JINR-P2-86-835 (1986).

[13] V.G.Kadyshevsky, D.V.Fursaev, JINR-P2-87-913 (1987); Sov.Phys.Dok1.34, p. 534 (1989).

[14] V.G.Kadyshevsky, V.N.Rodionov, Phys. Part. Nucl., 36, no.1, S34-S37 (2005).

[15] C.M.Bender and S.Boettcher, Phys. Rev. Lett., 80, p.5243 (1998).

[16] C.M.Bender, S.Boettcher and P.N. Meisinger, J. Math. Phys., 40, p.2210 (1999).

[17] A.Mostafazadeh, Int. J. Geom. Meth. Mod. Phys., 7, p.1191-1306 (2010); arXiv: 0810.5643 (2008).

[18] A.Khare, B.P.Mandal, Phys. Lett.A, 272, p.53 (2000).

[19] M.Znojil, G.Levai, Mod. Phys. Lett.A, 16, p.2273 (2001).

[20] A.Mostafazadeh, J. Phys.A 38 p.6657 (2005), Erratum-ibid. 38, p.8185 (2005).

[21] C.M.Bender, D.C.Brody, J.Chen, H.F.Jones, K.A.Milton, and M.C.Ogilvie, Phys. Rev.D, 74, p.025016 (2006). and see refs therein.

[22] C.M.Bender, K.Besseghir, H.F.Jones, and X. Yin, J.Phys.A, 42, p.355301,(2009); arXiv: 0906.1291 (2009).

[23] A.Khare, B.P.Mandal, Spl. Issue of Pramana J. Phys., 73, p.387 (2009).

[24] P.Dorey, C.Dunning, and R.Tateo, J. Phys A: Math. Theor., 34 p.5679 (2001).

[25] C.M.Bender, D.C.Brody, and H.F.Jones, Phys. Rev.D 70 p.025001 (2004)(2004); Erratum-ibid. 71 p.049901 (2005).

[26] C.M.Bender, S.F.Brandt, J.Chen, and Q.Wang, Phys. Rev.D, 71, p.065010 (2005). 
[27] C.M.Bender, H.F.Jones and R.J.Rivers, Phys. Lett.B, 625 p.333 (2005).

[28] C.M.Bender, S.Boettcher, Phys. Rev. Lett., 89, p.270401-1 (2002); Erratum-ibid. 92, p.119902 (2004).

[29] C.M.Bender, J.Brod, A.Refig, and M.Reuter, J. Phys. A: Math. Gen., 37. p.10139, (2004); quant$\mathrm{ph} / 0402026$ (2004).

[30] A.Mostafazadeh, J. Math Phys., 43 p.205 (2002); 43 p.2814 (2002); 43 p.3944 (2002).

[31] A.Mostafazadeh, A. Batal, J. Phys A: Math. Theor., 37, p.11645 (2004).

[32] A.Mostafazadeh, J. Phys A: Math. Theor., 36, p.7081 (2003).

[33] M.Mostafazadeh, Class. Q. Grav. 20, p.155 (2003).

[34] M.Mostafazadeh, Ann. Phys., 309, p.1 (2004).

[35] M.Mostafazadeh, F.Zamani, Ann. Phys., 321, p.2183; p.2210 (2004).

[36] M.Mostafazadeh, Int. J. Mod. Phys. A, 21, no.12 p.2553 (2006).

[37] F.Zamani, M.Mostafazadeh, J. Math. Phys., 50, p.052302 (2009).

[38] V.N.Rodionov. PT-symmetric pseudo-Hermitian relativistic quantum mechanics with maximal mass. arXiv:hep-th/1207.5463 (2012).

[39] V.N.Rodionov, G.A.Kravtsova, Mosc. Phys. Bull., 69, no.3, p.223 (2014).

[40] V.N.Rodionov, G.A.Kravtsova, Theor. Math. Phys., 182, p.100 (2016).

[41] V.N.Rodionov. Non-Hermitian PT-symmetric quantum mechanics of relativistic particles with the restriction of mass. arXiv:1303.7053 (2013).

[42] I.P.Volobuev, V.G.Kadyshevsky, M.D.Mateev, and M.R.Mir-Kasimov, Theor. Math. Phys., 40, p.363 (1979).

[43] V.N.Rodionov. On limitation of mass spectrum in non-Hermitian PT-symmetric models with the $\gamma_{5}$-dependent mass term. arXiv:1309.0231 (2013).

[44] V.N.Rodionov. Non-Hermitian PT-symmetric relativistic Quantum mechanics with a maximal mass in an external magnetic field. arXiv:1404.0503 (2014);

[45] V.N.Rodionov. Exact Solutions for Non-Hermitian Dirac-Pauli Equation in an intensive magnetic field. arXiv:1406.0383 (2014);

[46] I.M.Ternov, V.R.Khalilov, and V.N.Rodionov. textitInteraction of charged particles with intensive electromagnetic field. (Moscow State Univ. Press., Moscow, 1982).

[47] V.N.Rodionov, Phys. Scr., 90, p.045302 (2015).

[48] V.N.Rodionov. Int. J. Theor.Phys., 54, p.3907 (2015).

[49] V.N.Rodionov. Non-Hermitian PT-Symmetric Relativistic Quantum Theory in an Intensive Magnetic Field. In: Non-Hermitin Hamiltonians in Quantum Physica. Selected Contributions from the 15th International Conference on Non-Hermitian Hamiltonians in Quantum Physics, Palermo, Italy, 18-25 May 2015. Eds.: F.Bagarello, R.Passante, C.Trapani. (Springer Proc. Phys., 184, Springer, Switzerland, 2016) p.357.

[50] V.N.Rodionov, G.A.Kravtsova, Phys. Part. Nucl., 47, no.2, p.135 (2016); arXiv:1603.03063 (2016).

[51] V.N.Rodionov. Non-Hermitian PT-Symmetric Dirac-Pauli Hamiltonians with Real Energy Eigenvalues in the Magnetic Field. arXiv:1409.5412 (2014).

[52] V.N.Rodionov. Non-Hermitian PT-symmetric relativistic quantum theory in an intensive magnetic field. arXiv:1603.07443 (2016).

[53] V.N.Rodionov. Towards the detecting of pseudo-Hermitian anomalies for negative square masses neutrinos in intensive magnetic fields. arXiv:1603.08425 (2016).

[54] V.M.Lobashev, Nucl. Phys. A, 719, p.C153 (2003). 
[55] V.N.Aseev et al., Phys. Rev. D, 84, p.112003 (2011).

[56] V.N.Aseev, A.I.Belesev et al., Phys. At. Nucl., 75, p.464 (2012). 